\title{
Ca isotopes fractionation at thermodynamical equilibrium between solids (silicates, carbonates, oxides) from Density Functional Theory: functional matters, and rescaling is not recommended.
}

\section{MERLIN MÉHEUT}

CNRS Géosciences Environment Toulouse, Université de Toulouse, UPS, IRD, CNES

Presenting Author: merlin.meheut@get.omp.eu

The isotopic composition of $\mathrm{Ca}$ isotopes has found numerous applications in geology, environmental sciences or medicine. For those applications, the knowledge of the fractionation occurring between two different phases at thermodynamical equilibrium is of primary importance.

In this work, the fractionation of $\mathrm{Ca}$ isotopes at equilibrium has been computed between various solids: calcite, aragonite, lime $\mathrm{CaO}$, diopside, grossular. At $25^{\circ} \mathrm{C}$, the predicted fractionations w/r to calcite are ${ }^{\mathrm{VIII}} \mathrm{Ca}_{3} \mathrm{Al}_{2} \mathrm{Si}_{4} \mathrm{O}_{12}$ grossular $(+4 \%$ ) $>{ }^{\mathrm{VI}} \mathrm{CaO}(+2 \%)>{ }^{\mathrm{VIII}} \mathrm{CaMgSi}_{2} \mathrm{O}_{6}$ diopside $(0 \%)>{ }^{\mathrm{VIII}} \mathrm{CaCO}_{3}$ aragonite $(-3 \%)$.

Importantly, the result strongly depends on the chosen functional (with PZ, PBE, vdW-DF2, BLYP, BLYP + GrimmeD2 correction, and PBESOL functionals being tested). In terms of mineral-calcite fractionation at $25^{\circ} \mathrm{C}$, the choice of the functional induces variations of up to $1.3 \%$ for the same mineral.

We analyzed the contributions of the different vibrational modes, and attempted to correct our calculations for the error commited on vibrational frequencies. The common procedure of frequency rescaling, based on the computation of an average relative error $\langle\chi\rangle$ on frequencies, and on the uniform correction of all frequencies for this error, shows highly inefficient in the case of $\mathrm{Ca}$, as it further increases the dispersion of the results obtained by different functionals. $\mathrm{Ca}$ isotopes fractionation properties depend on low (below $400 \mathrm{~cm}^{-1}$ typically) frequency modes, which can show relative errors large with respect to experiment, and disconnected with higher frequency, uncontributing modes. A proper account of the relative contributions of the different modes permits to reconcile the results of all the gradient-corrected functionals.

A detailed analysis of the accuracy of the calculations suggests that the PBESOL functional is superior and more reliable than the others. It shows the smallest and more constant error on frequencies for any given material. It shows also most consistent for errors on structural, vibrational and fractionation properties between different materials. PBE shows comparably efficient, while the other functionals give important deviations in some cases. While not having been explicitly tested here, it is suggested from literature that the B3LYP functional might not be superior to PBESOL for this application. 